\title{
ON RINGS WITH PRIME CENTERS
}

\section{HAZAR ABU-KHUZAM}

\author{
Department of Mathematics \\ American University of Beirut \\ Beirut, Lebanon
} and

\section{ADIL YAQUB}

Department of Mathematics University of California at Santa Barbara Santa Barbara, California, U.S.A.

(Received December 4, 1992 and in revised form June 26, 1993)

\begin{abstract}
Let $R$ be a ring, and let $C$ denote the center of $R . R$ is said to have a prime center if whenever $a b$ belongs to $C$ then $a$ belongs to $C$ or $b$ belongs to $C$. The structure of certain classes of these rings is studied, along with the relation of the notion of prime centers to commutativity. An example of a non-commutative ring with a prime center is given.
\end{abstract}

KEY WORDS AND PHRASES. Commutativity, prime center, periodic ring, prime ring. 1991 AMS SUBJECT CLASSIFICATION CODE. 16 U80.

\section{INTRODUCTION.}

Throughout, $R$ is an associative ring, $N$ denotes the set of nilpotent elements of $R, C$ denotes the center of $R$, and $J$ denotes the Jacobson radical of $R$.

We introduce the notions of "prime centers" and "semiprime centers" as follows:

DEFINITIONS: A ring $R$ is said to have a prime center if whenever $a b \in C$ then $a \in C$ or $b \in C . R$ is said to have a semiprime center if whenever $x^{n} \in C$ then $x \in C$.

A ring $R$ is called periodic if for each $x$ in $R$, there exist distinct positive integers $m$ and $n$ for which $x^{m}=x^{n}$. The following lemma is known [1] and we omit its proof.

LEMMA 1. Let $R$ be a periodic ring. Then for each $x$ in $R$, there exists a positive integer $k=k(x)$ such that $x^{k}$ is idempotent.

Clearly, every commutative ring has a prime center and a prime center is a semiprime center. Our objective is to study the structure of certain classes of rings with prime centers, and the relation of this notion to commutativity. We also give an example of a noncommutative ring having a prime center.

2. MAIN RESULTS.

The following gives some basic properties of rings with prime or semiprime center.

BASIC LEMMA 2.

(a) Let $R$ be a ring having a semiprime center. Then $N \subseteq C$ and the idempotent elements of $R$ also belong to the center. 
(b) Let $R$ be a ring with identity 1 and having a prime center. Then the units of $R$ are central and hence $J \subseteq C$.

(c) Let $R$ be a prime ring having a semiprime center. If $e$ is an idempotent of $R$, then $e=0$ or $e=1$ (if $R$ has 1 ).

(d) Let $\left\{R_{\mathfrak{z}}, i \in \Gamma\right\}$ be a family of rings. If the direct sum $\underset{i \in \Gamma}{\sum^{\prime}} R_{\mathrm{t}}$ has a prime center, then $R_{t}$ has a prime center for each $i \in \Gamma$. Moreover, at most one $R_{t}$ is noncommutative.

PROOF. (a) Let $a$ be any nilpotent element in $N$. Then $a^{k}=0$ for some positive integer $k$, and hence $a^{k} \in C$. This implies that $a \in C$ since $R$ has a semiprime center. So $N \subseteq C$. Let $e$ be any idempotent element and $x$ any element of $R$. Then, $(e x-e x e) \in N$ and hence

$$
e(e x-e x e)=(e x-e x e) e .
$$

This implies that $e x=e x e$. Similarly $x e=e x e$, and hence $e$ belongs to the center of $R$.

(b) Let $u$ be any unit in $R$. Then $u u^{-1}=1 \in C$. This implies that $u \in C$ or $u^{-1} \in C$, and hence $u \in C$. If $a \in J$ then $a+1$ is a unit, and hence central. So $J \subseteq C$.

(c) Let $e$ be an idempotent element of $R$. By Lemma 2(a), $e$ is a central idempotent and hence

$$
e R(e x-x)=0 \text { for all } x \in R .
$$

This implies, since $R$ is a prime ring, that

$$
e=0 \text { or } e x=x \text { for all } x \in R .
$$

If $e \neq 0$, then $e x=x$ for all $x \in R$. Since $e \in C$, therefore $x e=x$ for all $x \in R$ also. Hence $e=1$.

(d) Let $C$ be the center of $\sum_{i \in \Gamma} R_{a}$ and $C_{\text {a }}$ be the center of $R_{1}$ for each $i \in \Gamma$. Let $a_{j}$ and $b$, be two elements of $R_{j}(j \in \Gamma)$ such that $a, b, \in C_{j}$. Let $\left\{a_{\mathrm{t}}\right\}$ and $\left\{b_{\mathrm{z}}\right\}$ be two elements in the direct $\operatorname{sum}, \sum_{\in} \cdot R$, such that

Then

$$
a_{\mathbf{s}}=\left\{\begin{array}{ll}
0 & \text { if } i \neq j \\
a, & \text { if } i=j
\end{array} \quad b_{\mathbf{i}}= \begin{cases}0 & \text { if } i \neq j \\
b, & \text { if } i=j\end{cases}\right.
$$

$$
\left\{a_{a}\right\} \cdot\left\{b_{i}\right\}= \begin{cases}0 & \text { if } i \neq j \\ a, b, & \text { if } i=j\end{cases}
$$

This implies that $\left\{a_{\mathrm{u}}\right\}\left\{b_{\mathrm{z}}\right\} \in C$ since $a_{\mathrm{j}}, b_{\mathrm{j}} \in C_{\mathrm{j}}$ (the center of $R_{\mathrm{j}}$ ). Hence $\left\{a_{\mathrm{i}}\right\} \in C$ or $\left\{b_{i}\right\} \in C$ since $\sum_{i \in \Gamma} R_{i}$ has a prime center. Therefore, $a_{j} \in C$, or $b_{\jmath} \in C_{\jmath}$. So $R$, has a prime center for each $j \in \Gamma$.

To complete the proof, suppose $R_{i}$ and $R_{j}$ are both noncommutative, $i \neq j$. Let $a_{i} \in R_{i} \backslash C_{\imath}, a, \in R_{j} \backslash C_{j}$ (where $C_{i}, C$, denote the centers of $R_{i}, R_{j}$, respectively). Let

$$
a_{k}=\left\{\begin{array}{ll}
0 & \text { if } k \neq i \\
a_{i} & \text { if } k=i
\end{array} \text { and } b_{k}=\left\{\begin{array}{ll}
0 & \text { if } k \neq j \\
a_{j} & \text { if } k=j
\end{array} .\right.\right.
$$

Then $\left\{a_{k}\right\}\left\{b_{k}\right\}=0 \in C$ while $\left\{a_{k}\right\} \notin C,\left\{b_{k}\right\} \notin C$, contradiction.

We now proceed to prove the main theorems.

THEOREM 1. Let $R$ be a periodic ring. Then $R$ is commutative if and only if $R$ has a semiprime center.

PROOF. If $R$ is commutative, then $R$ clearly has a semiprime center. Suppose that $R$ has a semiprime center and let $x$ be any element of $R$. Since $R$ is periodic, Lemma 1 implies that $x^{k}$ 
is idempotent for some positive integer $k$. Hence by Lemma $2(a), x^{k} \in C$. This implies that $x \in C$, since $R$ has a semiprime center.

THEOREM 2. Let $R$ be a prime ring with a semiprime center. Then $R$ is a domain.

PROOF. By Lemma 2(a), $N \subseteq C$. But the center of a prime ring has no nonzero zero divisors, and hence $N=\{0\}$. It is well known that a prime ring with $N=\{0\}$ is a domain.

THEOREM 3. Let $R$ be an Artinian ring with prime center, and $N$ the set of nilpotent elements. Then $N$ is an ideal and $\frac{R}{N}$ is a direct sum of fields.

PROOF. By Lemma 2(a), $N \subseteq C$; and this implies that $N$ is an ideal. Now $\frac{R}{N}$ is Artinian and reduced, so by the Wedderburn-Artin structure theory, $\frac{R}{N}$ is a (finite) direct sum of division rings. let $R_{1}$ be a division ring which is an internal direct summand of $\frac{R}{N}$ and let $\bar{e}=u+N$ be the identity element of $R_{1}$. Since idempotents may be lifted, there exists an idempotent $e \in R$ such that $\bar{e}=e+N$. By Lemma 2(a), $e \in C$.

Let $\bar{x}=x+N$ and $\bar{y}=y+N$ be inverses in $R_{1}$. Then $\overline{x y}=\bar{e}$, so $x y=e+v$ for some $v \in N$. Thus $x y \in C$, and hence $x \in C$ or $y \in C$. So $\bar{x}$ or $\bar{y}$ is central in $R_{1}$. But if one is, so is the other. Hence $R_{1}$ is commutative, and thus $R_{1}$ is a field. This proves the theorem.

COROLLARY 1. Let $R$ be a prime Artinian ring with identity 1 . If $R$ has a prime center, then $R$ is a field.

PROOF. Let $I$ be a nonzero ideal of $R$. Then $I$ is non-nilpotent, since $R$ is a prime ring. This implies that $I$ contains a nonzero idempotent element, since $R$ is Artinian ([2], Theorem 1.3.2). So, by Lemma 2(c), $1 \in I$ and hence $I=R$. This implies that $R$ is a simple ring. Hence, by Theorem $3, R$ is a field.

COROLLARY 2. Let $R$ be a semisimple Artinian ring. If $R$ is a prime center, then $R$ is isomorphic to a direct sum of fields.

PROOF. This follows at once from Theorem 3 , since $N=\{0\}$.

THEOREM 4. Let $R$ be a ring with identity and having a prime center. If for each $x \in R$, there is a monic polynomial $f=f_{x}$ with integer coefficients such that $f(x) \in C$, then $R$ is commutative.

PROOF. We will prove that if $x \in R$ with $f_{x}(x) \in C$, then $x \in C$. We proceed by induction on the degree $n$ of the polynomial $f_{x}$. If $n=1$, then $x \in R$ with $f_{x}(x)=x+a_{0} \in C$. This implies that $x \in C$. Suppose that the above statement is true for all $x \in R$ having a polynomial $f_{x}$ of degree $n$ with $f_{x}(x) \in C$. Let $y \in R$ with

$$
f_{y}(y)=y^{n+1}+a_{n} y^{n}+\cdots+a_{1} y+a_{0} \in C
$$

where $a_{0}, \cdots, a_{n}$ are integers. Thus

$$
y^{n+1}+a_{n} y^{n}+\cdots+a_{1} y \in C,
$$

and hence

$$
y\left(y^{n}+a_{n} y^{n-1}+\cdots+a_{2} y+a_{1}\right) \in C .
$$

If $y \notin C$, then $y^{n}+a_{n} y^{n-1}+\cdots+a_{1} \in C$ since $R$ has a prime center. So, $y$ has a polynomial $g$ of degree $n$ with $g(y) \in C$. Hence $y \in C$ by the induction hypothesis. This completes the proof of Theorem 4.

\section{EXAMPLE OF A NONCOMMUTATIVE RING WITH A PRIME CENTER.}

Choose $F$ to be an infinite field admitting an automorphism $\sigma$ of infinite order. Let $F[x, \sigma]$ 
be the skew polynomial ring of all polynomials $p(x)$ over $F$ such that $x^{n} a=\sigma^{n}(a) x^{n}$ for every $a \in F$ and every positive integer $n$. It is easy to verify that $F[x, \sigma]$ is a domain.

Let $R=F[x, \sigma] x$. We will show that $R$ is a noncommutative ring having a prime center. Let $C$ denote the center of $R$ and let $P(x)=a_{1} x+a_{2} x^{2}+\cdots+a_{n} x^{n}$ be any nonzero element in the center of $R$, it being assumed that $a_{n} \neq 0$. Now,

and

$$
\begin{aligned}
x P(x) & =x a_{1} x+x a_{2} x^{2}+\cdots+x a_{n} x^{n} \\
& =\sigma\left(a_{1}\right) x^{2}+\sigma\left(a_{2}\right) x^{3}+\cdots+\sigma\left(a_{n}\right) x^{n+1}
\end{aligned}
$$

$$
P(x) x=a_{1} x^{2}+a_{2} x^{3}+\cdots+a_{n} x^{n+1} .
$$

But $x P(x)=P(x) x$, since $P(x) \in C$. This implies that

$$
\sigma\left(a_{1}\right)=a_{1}, \sigma\left(a_{2}\right)=a_{2}, \cdots, \sigma\left(a_{n}\right)=a_{n} .
$$

Since $\sigma$ has an infinite order, there exists an element $a \in F$ such that

$$
\sigma^{n}(a) \neq a
$$

Now,

$$
\begin{aligned}
\operatorname{axP}(x) & =a x a_{1} x+a x a_{2} x^{2}+\cdots+a x a_{n} x^{n} \\
& =a \sigma\left(a_{1}\right) x^{2}+a \sigma\left(a_{2}\right) x^{3}+\cdots+a \sigma\left(a_{n}\right) x^{n+1} \\
& =a a_{1} x^{2}+a a_{2} x^{3}+\cdots+a a_{n} x^{n+1} \quad \text { by (3.1) }
\end{aligned}
$$

Also,

$$
\begin{aligned}
P(x) a x & =a_{1} x a x+a_{2} x^{2} a x+\cdots+a_{n} x^{n} a x \\
& =a_{1} \sigma(a) x^{2}+a_{2} \sigma^{2}(a) x^{3}+\cdots+a_{n} \sigma^{n}(a) x^{n+1} .
\end{aligned}
$$

But $a x P(x)=P(x) a x$, since $P(x) \in C$. This implies that

$$
a a_{1}=a_{1} \sigma(a), a a_{2}=a_{2} \sigma^{2}(a), \cdots, a a_{n}=a_{n} \sigma^{n}(a)
$$

Since $F$ is a field and $a_{n} \neq 0$, the last of these equations contradicts (3.2). Hence $P(x)=0$, and the center $C=\{0\}$. Thus, $R=F[x, \sigma] x$ is a domain with center $\{0\}$. This implies that $R$ has a prime center. For, if $P(x) q(x) \in C=\{0\}$, then $P(x) \cdot q(x)=0$. This implies that $P(x)=0 \in C$ or $q(x)=0 \in C$, since $R$ is a domain. $R$ is clearly a noncommutative ring $(x a=\sigma(a) x \neq a x)$.

ACKNOWLEDGEMENT. We would like to express our gratitude and indebtedness to the referee for the helpful suggestions made, which resulted in improving the results of this paper.

\section{REFERENCES}

1. BELL, H.E., Some commutative results for periodic rings, Acta. Math. Acad. Sci. Hungar., 28 (1976), 279-283.

2. HERSTEIN, I.N., Noncommutative Rings, The Carus Math. Monographs, John Wiley and Sons, Inc., 1968. 


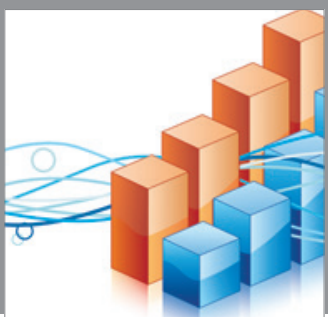

Advances in

Operations Research

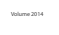

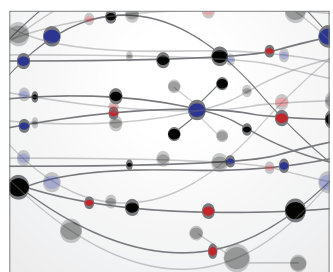

\section{The Scientific} World Journal
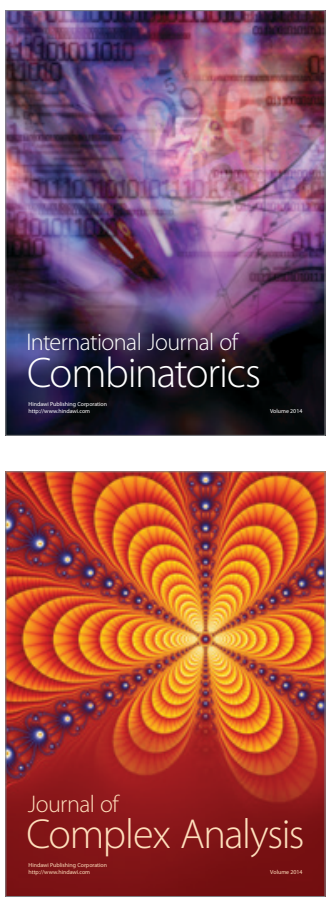

International Journal of

Mathematics and

Mathematical

Sciences
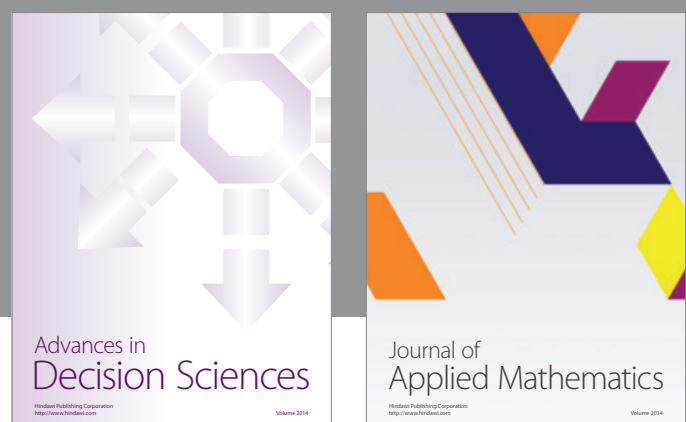

Journal of

Applied Mathematics
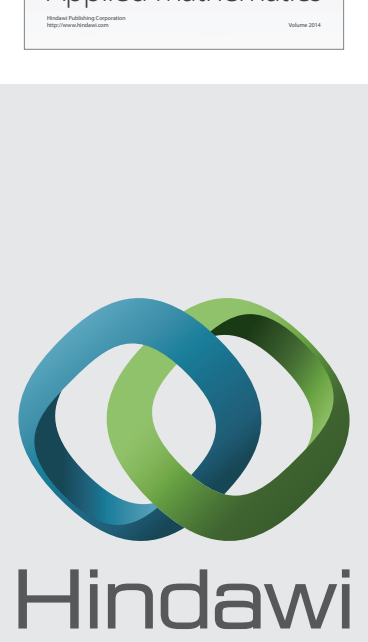

Submit your manuscripts at http://www.hindawi.com
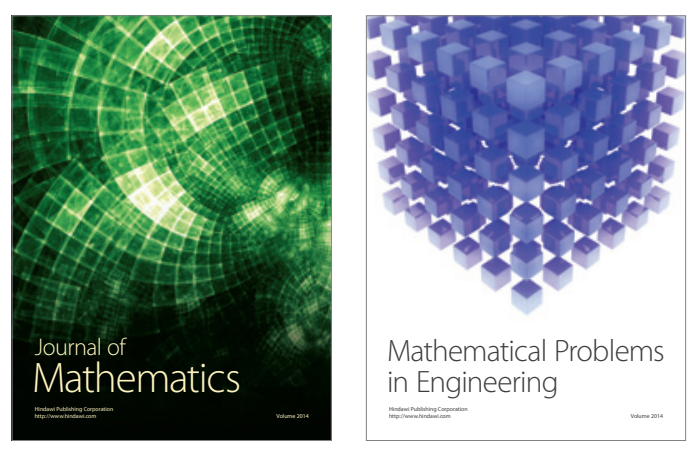

Mathematical Problems in Engineering
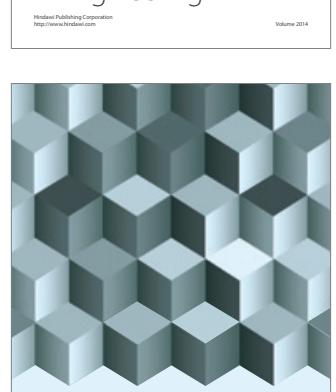

Journal of

Function Spaces
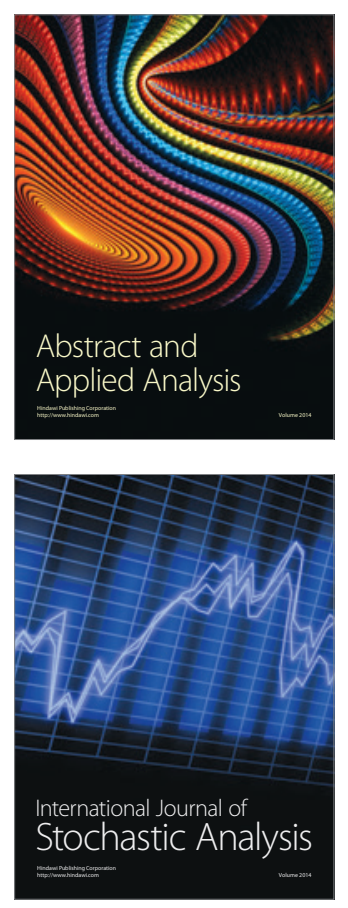

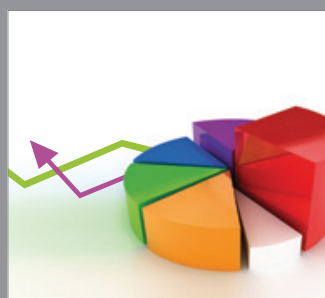

ournal of

Probability and Statistics

Promensencen
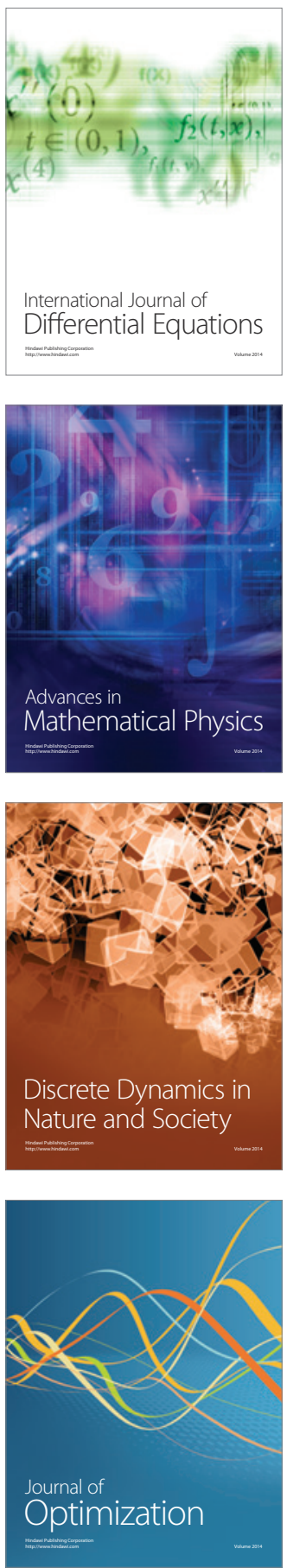